SHORT REPORT

\title{
Difficulty in diagnosing chronic meningitis caused by capsule-deficient Cryptococcus neoformans
}

\author{
Y Sugiura, M Homma, T Yamamoto
}

J Neurol Neurosurg Psychiatry 2005;76:1460-1461. doi: 10.1136/jnnp.2004.052662

We report a case of chronic meningitis due to capsuledeficient Cryptococcus neoformans which could not be diagnosed by routine morphological and immunological cerebrospinal fluid (CSF) examination. Repeated CSF examination and culture did not disclose the fungal body, and the cryptococcal antigen tests were always negative. Culture of ventricular fluid showed non-encapsulated cultured cells which were stained positively by indirect immunofluorescence using diluted sera from another patient diagnosed with cryptococcal meningitis. Inoculation of dispersed colonies into mice resulted in encapsulated $C$ neoformans. It is important to suspect capsule-deficient $C$ neoformans when the conventional diagnostic approaches fail to identify the organism or antigens.

$\mathrm{T}$ he form of Cryptococcus neoformans which infects humans typically possesses a thick capsule and routine morphological diagnosis depends on that unique feature. However, we encountered a case of chronic meningitis caused by capsule-deficient $C$ neoformans. Because it was so different from the classical form, this posed a diagnostic challenge since conventional approaches were not informative. Here, we report the case together with a discussion of the pitfalls in reaching a diagnosis with this particular form of $C$ neoformans.

\section{CASE REPORT}

A 42 year old man working as a farmer was admitted to a local hospital on July 6, 1992 after having had an episode of loss of consciousness following tonic-clonic convulsion. There was a history of chronic heavy alcohol consumption. A low grade fever and general fatigue preceded the event by several weeks. His cerebrospinal fluid (CSF) cell count from lumbar puncture was increased to $168 / \mathrm{mm}^{3}$ (lymph:polys 1:1) and CSF protein was $116 \mathrm{mg} / \mathrm{dl}$. Aciclovir, ceftriaxone, and sulbenicillin sodium were started intravenously. However, his symptoms did not improve and 1 month later isoniazid, rifampicin, ampicillin, and minocycline hydrochloride were started. On October 26, he had further fits and deterioration. He was transferred on December 16 to a university general hospital because of the difficulty in identifying the cause of meningitis. CSF findings were pleocytosis $\left(74 / \mathrm{mm}^{3}\right)$ and increased protein $(151 \mathrm{mg} / \mathrm{dl})$. Herpes simplex virus, varicella zoster virus antibodies, and cryptococcal antigen were negative in CSF. Despite institution of antituberculous regimens again, his clinical course slowly deteriorated and on April 16, 1993, he was transferred to our hospital.

On admission, he was moderately debilitated, his temperature was $37.1{ }^{\circ} \mathrm{C}$, and his blood pressure was 162/ $100 \mathrm{~mm} \mathrm{Hg}$. Thereafter, he was conscious most of the time and could express himself, but he was markedly disoriented. He could stand and walk in an unsteady, ataxic manner and Romberg's test was positive. Deep tendon reflexes were active throughout and the planter response was bilaterally extensor. The forced grasping and sucking reflexes were seen. Sense of position was diminished in his bilateral lower extremities. The cranial nerves were normal. His muscle power was not fully tested but showed no apparent weakness. Neither stiff neck nor the Kernig's sign were observed.

A lumbar puncture yielded clear, colourless fluid with an opening pressure of $140 \mathrm{~mm} \mathrm{CSF}$, an increased cell count $\left(69 / \mathrm{mm}^{3}, 78 \%\right.$ monocytes $)$, elevated protein (221 mg/dl), and low sugar $(8 \mathrm{mg} / \mathrm{dl}, \mathrm{CSF} / \mathrm{blood}$ glucose ratio $8.2 \%)$. Gram staining, Indian ink preparations, CSF cytology with ZiehlNeelsen staining, cultures for fungi, and cryptococcal antigen were all negative, and CSF examinations for Mycobacterium tuberculosis by culture and the polymerase chain reaction method were also negative. Full blood count showed RBC $4.10 \mathrm{million} / \mathrm{mm}^{3}$, platelets $75000 / \mathrm{mm}^{3}$, WBC $6100 / \mathrm{mm}^{3}$, lymphocytes $2257 / \mathrm{mm}^{3}$, CD4 $45.4 \%$, and CD8 $23.1 \%$. Laboratory studies revealed moderately impaired liver functions (GOT 35 IU/l, GPT 16 IU/l, LDH 443 IU/l, $\gamma$-GTP 63 IU/l, cholinesterase $185 \mathrm{IU} / \mathrm{l})$, total serum protein $7.4 \mathrm{~g} / \mathrm{dl}$, and albumin 3.9 g/dl. Fasting blood sugar was $97 \mathrm{mg} / \mathrm{dl}$. Hepatitis B surface antigen, hepatitis C antibody, and HIV antibody were negative. CT brain scan with contrast showed a small, circumscribed enhancing area at the right side of the basal cistern, suggesting the presence of a small granuloma (fig lA). On MRI T2 image, a circumscribed high signal intensity area was observed in the right ventral caudoputamen (fig 1B).

The patient complained of nausea. As an endoscopic examination had disclosed rather vulnerable looking oesophageal varices, endoscopic sclerosing therapy was performed on several occasions. A liver biopsy indicated advanced cirrhosis. Following generalised epileptic status which lasted intermittently for several hours, the patient became stuporous.

CSF examinations, repeated several times, always disclosed lymphocytic pleocytosis $\left(195 / \mathrm{mm}^{3}\right)$ without atypical cells, increased protein $(99 \mathrm{mg} / \mathrm{dl})$, and low glucose $(48 \mathrm{mg} / \mathrm{dl}$, $\mathrm{CSF} / \mathrm{blood}$ ratio $18 \%$ ), but Indian ink preparations, ZiehlNeelsen staining, cultures for fungi, and cryptococcal antigen were always negative. The differential diagnoses of cryptococcal meningitis, tuberculous meningitis, and meningeal carcinomatosis were considered. We highly suspected cryptococcal meningitis because there were no findings attributable to pulmonary tuberculosis and the antituberculous agents were not effective, and CSF cytology did not show carcinomatous cells. Therefore, an Ommaya reservoir was placed with the catheter tip inserted into the right frontal horn, both to study the ventricular fluid and to administer amphotericin B into the ventricle. However, cryptococcal antigen in the ventricular fluid via the Ommaya reservoir was reported to be equivocally positive. When the ventricular fluid was cultured on Sabouraud dextrose agar, milk-white colonies were seen after several days. The Indian ink preparation showed small round, bright spots with occasional

Abbreviations: CSF, cerebrospinal fluid 

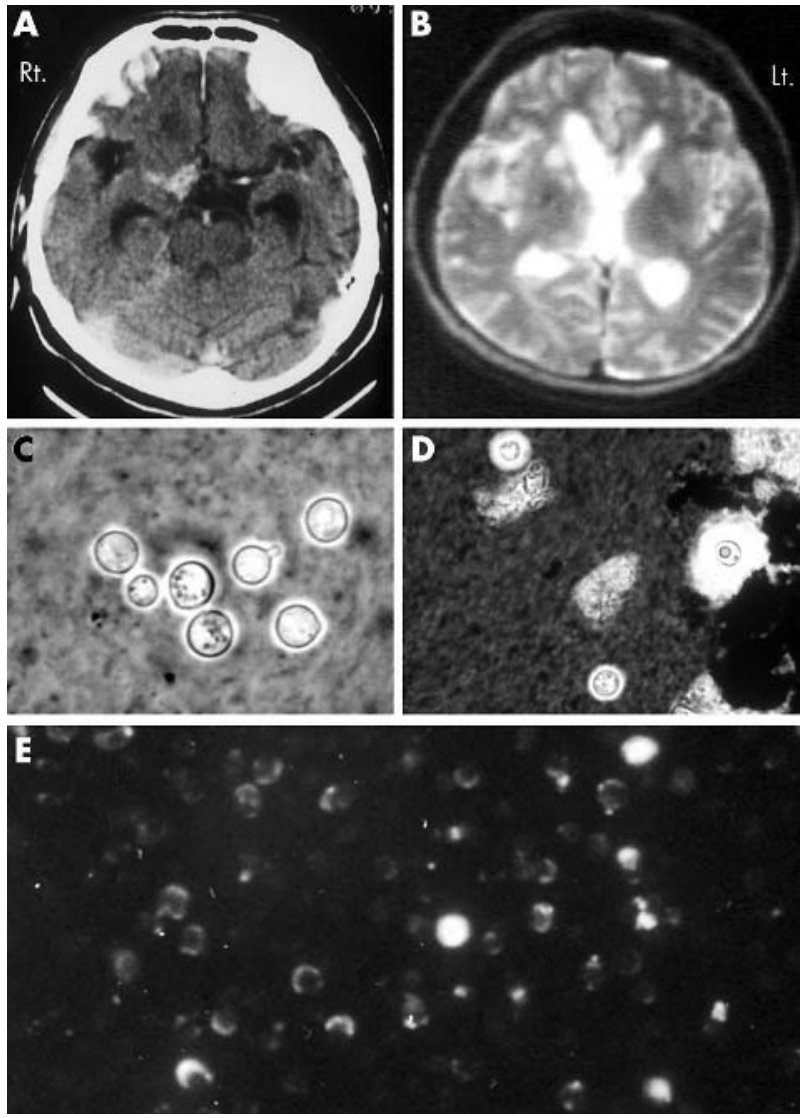

Figure 1 Brain CT with dye enhancement showed a small intensification at the right side of the basal cistern, suggesting the presence of a small granuloma (A). Brain MRI T2 image demonstrated a circumscribed high signal intensity area in the right ventral caudoputamen, suggesting the presence of inflammation (B). Small round, bright spots with budding appeared in the Indian ink preparation of the milk-white colonies which were cultured from ventricular fluid on Sabouraud dextrose agar (C). C neoformans with the classical appearance of thick walls was present in the ascites and brain 2 weeks after inoculation of the dissolved colonies into ICR mice (D). The nonencapsulated cultured cells were stained positively by indirect immunofluorescence using diluted sera from another patient diagnosed with cryptococcal meningitis (E).

budding (fig IC). Inoculation of dispersed colonies into ICR mice resulted in the classical appearance of $C$ neoformans with thick walls in their ascites and also in their brains after 2 weeks (fig 1D). Moreover, the non-encapsulated cultured cells were stained positively by indirect immunofluorescence using diluted sera from another patient diagnosed with cryptococcal meningitis (fig $1 \mathrm{E}$ ). The 23 carbohydrate assimilation, urease, nitrate, and cycloheximide sensitivity tests (Yeast Biochemical Card system, BioMerieux Vitek, Hazelwood, MO) were consistent with $C$ neoformans. A diagnosis of chronic meningitis caused by capsule-deficient $C$ neoformans was established and gradually increasing intravenous (20 mg/day) and intraventricular $(0.25 \mathrm{mg} /$ day $)$ doses of amphotericin B and oral flucytosine ( $10 \mathrm{~g} /$ day) were started. The CSF findings disclosed a decreased cell count $\left(2 / \mathrm{mm}^{3}\right)$, protein $(45 \mathrm{mg} / \mathrm{dl})$, and elevated sugar $(60 \mathrm{mg} / \mathrm{dl}$, CSF/blood glucose ratio $40.8 \%$ ). However, unfortunately the patient developed hepatorenal syndrome with subsequent multi-organ failure and death.

\section{DISCUSSION}

In this case, the typical encapsulated fungal body was not observed in or cultured from the CSF. Moreover, cryptococcal antigen was repeatedly negative by latex agglutination assay of the CSF. A diagnosis of chronic meningitis caused by capsule-deficient $C$ neoformans was established by indirect immunofluorescence staining and formation of the thick capsule after inoculation into the murine peritoneal cavity. In fact, only a few cases of cryptococcal meningitis caused by the capsule-deficient form have been reported. ${ }^{12}$

Capsule-deficient Cryptococcus produces sufficient capsule to inhibit phagocytosis when incubated with human lung tissue in vitro. ${ }^{3}$ In our case, the capsule-deficient type did form thick capsules in 2 weeks when inoculated into the murine peritoneal cavity. The capsule-deficient $C$ neoformans elicited an intense inflammatory response characterised by early suppuration and phagocytosis, followed by a marked histiocytic and fibroblastic reaction, but with limited spread of infection compared to the capsulated yeast form. ${ }^{4}$ Presumably, the brain MRI and CT scan showed a focus of parenchymatous inflammation and granuloma in our case. Meningitis due to capsule-deficient $C$ neoformans has previously been reported in AIDS patients (isolated from CSF) and in Japanese non-AIDS patient (isolated from ventricular fluid). ${ }^{12}$ The difference in these cases suggests that the host immune response influences the spread of capsule-deficient $C$ neoformans. Similarly, in our case repeated CSF examinations by lumbar puncture failed to detect yeast forms. The susceptibility of the capsule-deficient form to phagocytosis is probably the major reason why repeated CSF cultures failed to grow Cryptococcus, as it was quickly engulfed by macrophages on its way to the lumbar CSF space.

The repeated cryptococcal antigen tests of CSF were always negative. The sensitivity of the antigen test was $76 \%$ and its specificity was $98.9 \%$ for encapsulated Cryptococcus, however capsule deficiency would make the detection of cryptococcal antigen rather difficult, since the latex agglutination test for cryptococcal antigens detects solute capsular polysaccharide antigens with the antibody fixed to latex particles. ${ }^{5}$

It is important to consider the possibility of this capsuledeficient form when the presence of cryptococcal meningitis is strongly suspected, keeping in mind that the conventional diagnostic approaches fail to identify both the organism and the antigens.

\section{Authors' affiliations}

Y Sugiura, M Homma, T Yamamoto, Department of Neurology, Fukushima Medical University School of Medicine, Fukushima, 9601295 Japan

Competing interests: none declared

Correspondence to: Dr Yoshihiro Sugiura, Department of Neurology, Fukushima Medical University School of Medicine, 1 Hikarigaoka, Fukushima, 960-1295 Japan; sugiura@fmu.ac.jp

Received 26 August 2004

Revised version received 11 February 2005

Accepted 15 February 2005

\section{REFERENCES}

1 Ohara $\mathrm{Y}$, Nakamura S, Otomo $\mathrm{H}$, et al. A case with cryptococcal meningitis caused by capsule-deficient Cryptococcus neoformans. Neurol Med 1977;7:76-9

2 Bottone EJ, Toma M, Johansson BE, et al. Capsule-deficient Cryptococcus neoformans in AIDS patients. Lancet 1985;8425:400.

3 Farhi F, Bulmer GS, Tacker JR. Cryptococcus neoformans IV. The not-soencapsulated yeast. Infect Immun 1970;1:526-31.

4 Farmer SG, Komorowski RA. Histologic response to capsule-deficient Cryptococcus neoformans. Arch Pathol 1973;96:383-7.

5 Tanaka K, Kohno S, Miyazaki T, et al. The Eiken Latex test for detection of a cryptococcal antigen in cryptococcosis. Comparison with a monoclonal antibody-based latex agglutination test, Pastorex cryptococcus. Mycopathologia 1994;127:131-4. 